\title{
Functional Vessel Density in the First Month of Life in Preterm Neonates
}

\author{
JULIA KROTH, KATHRIN WEIDLICH, STEPHAN HIEDL, CLAUDIA NUSSBAUM, FRANK CHRIST, AND \\ ORSOLYA GENZEL-BOROVICZÉNY
}

\begin{abstract}
Clinic of Dermatology and Allerology [J.K.], University of Munich, 80337 Munich, Germany; Department of Internal Medicine [K.W.], General Hospital Munich, 80804 Munich, Germany; Department of Gynecology and Obstetrics [O.G.B., S.H., C.N.], Division of Neonatology, University Children's Hospital University of Munich, 80337 Munich, Germany; Clinic for Anesthesiology [F.C.], University of Munich, Grosshadern 81366 Munich, Germany
\end{abstract}

\begin{abstract}
Changes in microcirculation have been recognized as central to many disease processes. The aim of this study was to evaluate factors, which influence the microcirculation of the skin during the first month of life in premature infants. Red blood cell (RBC) velocity, vessel diameter, and functional small vessel density (FSVD) were measured daily for the first $30 \mathrm{~d}$ on the upper arm in preterm infants with gestational age $<30 \mathrm{wk}$. Orthogonal polarization spectral (OPS) images were analyzed off-line with the CapiScope-Image program. In 25 infants, FSVD decreased significantly from week $1\left(\right.$ mean \pm SD $\left.236 \pm 33 \mathrm{~cm} / \mathrm{cm}^{2}\right)$ to week $4(207 \pm 30$ $\mathrm{cm} / \mathrm{cm}^{2}$ ) and correlated directly with $\mathrm{Hb}$ levels and incubator temperature. Vessel diameters and RBC velocity did not change significantly, nor did clinical parameters such as blood pressure, heart rate or body temperature. Microvascular parameters were not dependent on gestational or postnatal age. The microcirculation of the skin might be an easily accessible window to obtain better understanding of circulatory changes in the postnatal period. Our data are essential as basis for further studies in this field. $\mathrm{Hb}$ levels and possible incubator temperatures have a substantial influence on functional small vessel density and therefore need to be taken in account.
\end{abstract}

(Pediatr Res 64: 567-571, 2008)

$\mathrm{D}$ isturbances of the microcirculation play a key role in many disease states (1-4). The recent development of new technologies has helped to investigate these changes in adult patients with sepsis (5-7). Previous studies have shown that parameters of microcirculation such as microvessel diameter, red blood cell velocity, and functional small vessel density (FSVD) can be measured in the skin of term and preterm infants by Orthogonal Polarization Spectral (OPS) imaging in the first week of life (8) and that FSVD increases after elective blood transfusion in anemic neonates (9). The microcirculation of the skin plays an important role in maintaining a constant body temperature and in regulating the fluid balance $(10,11)$. Adequate function of the microcirculation is a prerequisite for tissue nutrition and oxygen supply (12). The microcirculation of the skin in neonates differs in several aspects from that of an adult. The regular architecture has been found to be poorly developed in the newborn (13). At birth, the skin shows a disorderly capillary network and no papillary loops in almost all areas, except the palms, soles, and nail

Received March 11, 2008; accepted June 10, 2008.

Correspondence: Orsolya Genzel-Boroviczény, M.D., Maistr. 11, 80337 Munich, Germany; e-mail: genzel@med.uni-muenchen.de folds. The skin is richly supplied by a dense subepidermal plexus demonstrating relatively little regional variation (14).

Functional capillary density (FCD) is one of the parameters that delineate the microcirculation. FCD is defined as the length of red cell-perfused capillaries per observation area and is given as $\mathrm{cm} / \mathrm{cm}^{2}$. FCD has been used as an indicator of the quality of tissue perfusion (15). In neonates capillary vessels, arterioles, and venules cannot clearly be differentiated in the OPS images, so that the expression FSVD is used.

The aim of the study was to determine whether tissue perfusion can be monitored reliably by OPS imaging in the premature infant in the first month of life and to see if very low gestational age or postnatal age have any influence on the imaging.

\section{METHODS}

Imaging. OPS imaging produces high-contrast microvascular images that are obtained from the absorption of light $(548 \mathrm{~nm})$ by the $\mathrm{Hb}$ in the blood without the use of fluorescent dyes. A virtual light source is created at a depth of $1 \mathrm{~mm}$ within the tissue using special optics. The reflected light yields an image of the illuminated vessel in negative contrast with a resolution of 1 pixel $\cong 1 \mu \mathrm{m}$. Only vessels perfused with red blood cells can be seen. The method has been validated for quantitative measurements of microcirculatory parameters in an animal model against intravital fluorescence microscopy. The use of OPS imaging in humans is possible without side effects $(16,17)$.

Calculation of vessel diameter, red blood cell velocity and functional small vessel density. Images were created using the CytoscanTM A/R, stored on videotape and analyzed off-line. Vessel diameter (Diam), red blood cell velocity (RBC vel), and FSVD were assessed with the CapiScope-Image program. The best video sequence with the least movement artifacts for at least $10 \mathrm{~s}$ was selected for each day and Diam and RBC vel were measured in 3 to 5 vessels per observation area and FSVD was calculated.

To measure the diameter, a vessel is selected from the image on the video screen and a line is placed in the vessel. The computer calculates the diameter from 1 pixel perpendicular lines placed over the whole length of the drawn line, based on the gray level change. RBC vel is measured using a spatial correlation technique, which requires a 10 -s video sequence in which the tissue reveals little movement. A line is drawn along the vessel and the image of the vessel is projected onto a 1 pixel thick line. The gray profile along the line is taken for each field every 1/50th of a second. The comparison is performed by calculating the correlation coefficient for every possible shift of the previous gray level profile relative to the new profile. The shift, which produces the highest correlation, indicates the distance that the pattern traveled between the two gray level profile measurements. Since the time lapse between the two gray level profiles is known, the velocity can be

Abbreviations: Diam, Vessel diameter; FCD, functional capillary density; FSVD, functional small vessel density; NIRP, near infrared photoplethysmography; OPS, orthogonal polarization spectral; RBC vel, red blood cell velocity 
calculated. Vessel density is defined as the length of RBC-perfused vessels per observation unit area and is given as $\mathrm{cm} / \mathrm{cm}^{2}$. For this article, the expression "vessel density" refers to perfused vessels (FSVD). In neonates due to the small size of vessels, arterioles, capillaries, and post capillary venules cannot be clearly distinguished, so calculations were done in vessels with diameters ranging from 6 to $24 \mu \mathrm{m}$ (CapiScope: www.kktechnology.com/ help/capiscope.html).

Quantitative measurement of RBC vel, FSVD and Diam using OPS imaging all have been validated against intravital microscopy in the hamster dorsal skin fold model, the rat liver and human sublingual tissue $(18,19)$. The new CapiScope software was validated against the previously used analysis system Cap Image (20).

Subjects. The study was approved by the ethics committee of the medical faculty of Ludwig-Maximilian University Munich. Parental consent was sought before measurements. OPS imaging was performed from day 3 to day 30 of life in preterm infants with a gestational age $<30 \mathrm{wk}$. Premature infants were nursed in incubators at ambient temperature.

Measurements were attempted every day at the same time and started when the infants settled down. The recording was done over 5 to $30 \mathrm{~min}$ to obtain as many sequences as possible of high quality with duration of at least $10 \mathrm{~s}$. The probe was placed in the incubator next to the upper arm near the axilla with the least possible contact to avoid pressure against the skin. In our previous studies, we have found that the best images were obtained from the skin of the inner upper arm. This area of the skin is less covered with lanugo hair, which may interfere with the imaging, and is sparsely affected by movement artifacts $e . g$., from breathing (8). Lubricating the skin with normal saline to increase the contact surface of skin and probe improved the imaging, in particular concerning sharpness and contrast.

Blood pressure, heart rate, incubator, body core and skin temperature were recorded at the time of measurement and $\mathrm{Hb}$ levels were measured daily.

To see if very low gestational age influences microcirculatory parameters we compared the most "mature" infants with a gestational age $>28$ wk with the least mature infants with a gestational age $<26 \mathrm{wk}$.

Statistics. Data are presented as mean for parametric data and as median for nonparametric data and $95 \%$ confidence interval (CI). Data were analyzed using repeated measures ANOVA followed by the paired Student $t$ test for comparison within the group and the unpaired Student $t$ test for comparison between preterm infants $<26$-wk gestational age and preterm infants $>28$-wk gestational age. The Mann-Whitney nonparametric test was used for nonparametric data. For correlation a Spearman rank order correlation and a linear regression (Pearson) was applied. The level of significance was set at $p<0.05$ and by a repeated $t$ test at $p<0.01$. All calculations were done with GraphPad Prism 4.0 for Windows XP (GraphPad Software Inc, San Diego CA, 2003).

\section{RESULTS}

We were able to obtained images for the first $30 \mathrm{~d}$ from 25 preterm infants (median [95\% CI]: gestational age of 28 [26.7-28.4] wk; birth weight 900 [808-993] g). Clinical data of the infants are shown in Table 1. Six infants received packed red blood cell transfusion during the study period at a median age of $21 \mathrm{~d}$. To calculate changes over time, results from $3 \mathrm{~d}$ were pooled for each week (days $6-8$ for week 1 , days $13-15$ for week 2 , days 20-22 for week 3 and days 27-29 for week 4). FSVD decreased significantly from the first week to the fourth week (Table 2, Fig. 1). FSVD correlated directly with $\mathrm{Hb}$ levels ( $r=0.76$ with $98 \%$ CI [0.540.86 ], $p=0.0001$ ) (Fig. 2). There was an inverse correlation
Table 2. Functional small vessel density (FSVD), red blood cell velocity (RBC vel), and vessel diameter (Diam) [mean $\pm S D$ and $95 \%$ confidence interval] for the 25 preterm infants

\begin{tabular}{lcccc}
\hline & Wk 1 & Wk 2 & Wk 3 & Wk 4 \\
\hline FSVD $\left(\mathrm{cm} / \mathrm{cm}^{2}\right)$ & $236 \pm 33$ & $219 \pm 33$ & $212 \pm 30$ & $207 * \pm 30$ \\
& $219-254$ & $205-234$ & $198-225$ & $191-223$ \\
RBC vel $(\mu \mathrm{m} / \mathrm{s})$ & $344 \pm 84$ & $338 \pm 60$ & $314 \pm 65$ & $314 \pm 54$ \\
& $299-388$ & $312-364$ & $284-343$ & $284-344$ \\
Diam $(\mu \mathrm{m})$ & $14.6 \pm 2.0$ & $14.4 \pm 2.4$ & $13.8 \pm 2.7$ & $14.2 \pm 1.3$ \\
& $13.5-15.6$ & $13.4-15.5$ & $12.6-15.0$ & $13.5-14.9$ \\
\hline
\end{tabular}

$* p=0.0028$ week 1 vs week 4 (paired Student $t$ test).
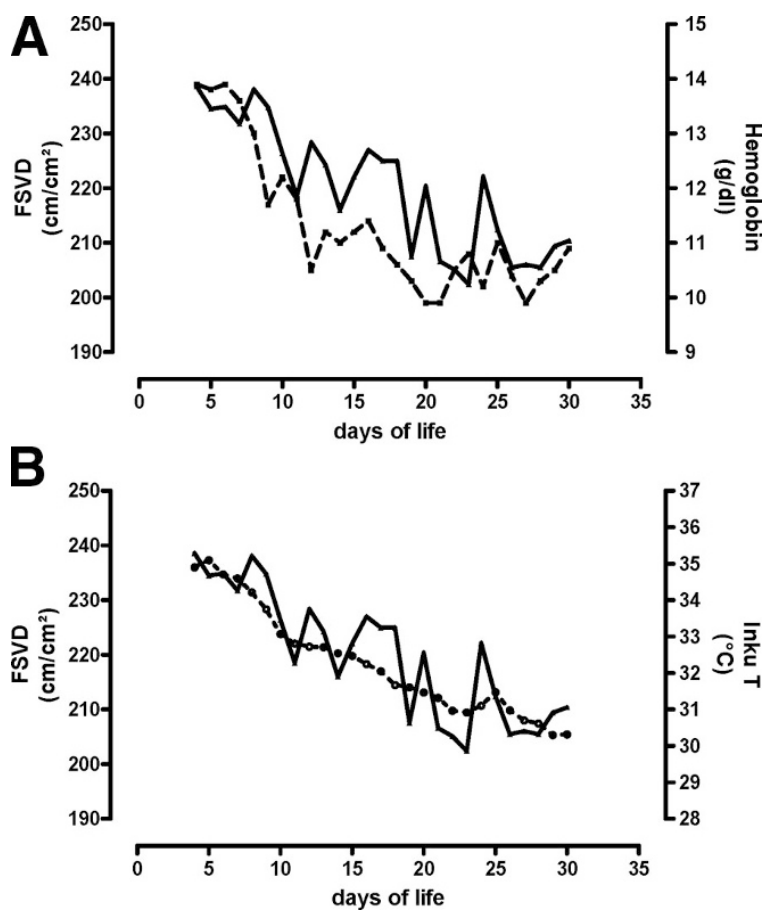

Figure 1. Correlation of $\mathrm{Hb}$ with FSVD in 25 preterm infants. Hb levels $(A,--)$, incubator temperature $(B, \ldots)$ and FSVD $(-)$ decreased significantly during the first month of life. Incubator temperature decreased linearly whereas Hb levels displayed "peaks" due transfusions. The curve for FSVD showed similar peaks.

between FSVD and heart rate $(r=-0.62$ with a 95\% CI $[-0.81$ to -0.32$], p<0.001)$ and systolic blood pressure $(r=$ -0.78 with a $95 \%$ CI $[-0.78$ to -0.24$], p=0.0021)$. FSVD did not correlate with body temperature or skin temperature at the area of measurement but with incubator temperature $(r=$ 0.87 with $98 \%$ CI [0.74-0.94], $p=0.0001$ ) (Fig. 2). Vessel diameter ranged from 7 to $24 \mu \mathrm{m}$, RBC vel from 172 to 727 $\mu \mathrm{m} / \mathrm{s}$ with no significant change during the first $30 \mathrm{~d}$ of life.

Table 1. Clinical data of the 25 preterm infants as median (95\% confidence interval)

\begin{tabular}{|c|c|c|c|c|}
\hline & Wk 1 & Wk 2 & Wk 3 & Wk 4 \\
\hline Weight $(\mathrm{g})$ & $836(695-976)$ & $942(843-1041)$ & $1048(912-1185)$ & $1140(964-1317)$ \\
\hline Systolic BP (mm Hg) & $53(46-59)$ & $57(53-60)$ & $59(54-63)$ & $58(53-63)$ \\
\hline Diastolic BP (mm Hg) & $30(25-35)$ & $35(32-39)$ & $33(31-36)$ & $34(29-38)$ \\
\hline MAP (mm Hg) & $39(34-43)$ & $43(40-47)$ & $41(38-44)$ & $42(38-46)$ \\
\hline Heart rate (beats/min) & $151(141-160)$ & $163(158-169)$ & $162(157-167)$ & $165(159-170)$ \\
\hline Hemoglobin (g/dl) & $13.6(11.8-15.4)$ & $11.0(10.3-11.6)$ & $9.9(9.2-10.6)$ & $10.3(9.2-11.3)$ \\
\hline Infant temperature $\left(\mathrm{C}^{\circ}\right)$ & $37(36.8-37.2)$ & $36.9(36.8-37.0)$ & $36.9(36.7-37.0)$ & $36.9(36.7-37.1)$ \\
\hline Incubator temperature $\left(\mathrm{C}^{\circ}\right)$ & $34.2(33.1-35.3)$ & $32.5(31.8-33.2)$ & $31(30.4-31.5)$ & $30.3(29.8-30.8)$ \\
\hline
\end{tabular}



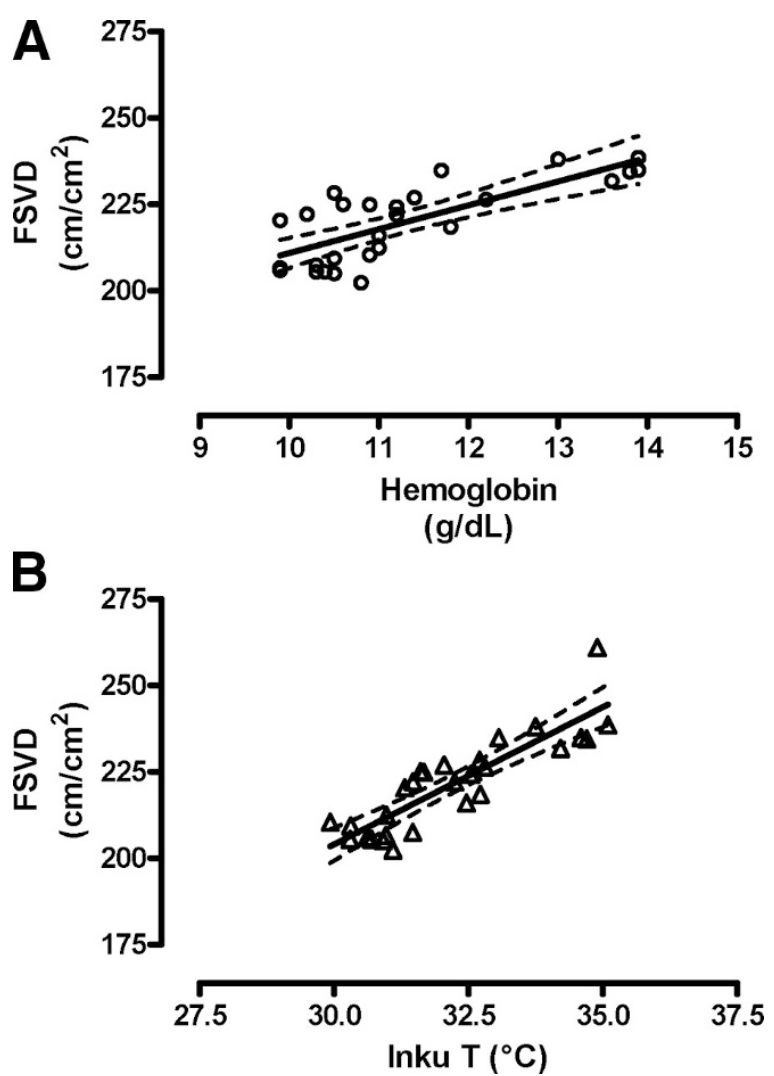

Figure 2. Correlation of $(A) \mathrm{Hb}$ and $(B)$ incubator temperature with FSVD. Both $\mathrm{Hb}$ levels and incubator temperature correlated directly with FSVD.

RBC vel correlated with Hb levels $(r=0.5$ with a $95 \%$ CI [0.14-0.74], $p=0.0083)$ and inversely with mean systolic blood pressure ( $r=-0.6$ with a $95 \%$ CI $[-0.8$ to -0.29$], p=$ 0.0009). Clinical variables, such as blood pressure, heart rate or body temperature did not change significantly. Hb levels decreased significantly over time $(p<0.0001)$. Gestational age had no influence on microcirculatory parameters.

\section{DISCUSSION}

We have shown that during the first month of life gestational or postnatal age do not influence microcirculatory parameters of the skin in preterm infants, but FSVD and RBC vel correlate with $\mathrm{Hb}$ levels. Using the same instrumentation sublingually, Nadeau and Groner observed a decline in vessel density in three severely anemic adults. They used the OPS imaging to calculate $\mathrm{Hb}$ levels by measuring the OD of the vessels and found a very good correlation (21). In our previous study packed red blood cell transfusion in anemic preterm infants increased FSVD $2 \mathrm{~h}$ after the transfusion with an additional rise $24 \mathrm{~h}$ later (9), but FSVD and $\mathrm{Hb}$ levels did not correlate. This apparent contradiction might be explained by the additional increase in FSVD $24 \mathrm{~h}$ after the transfusion without change in hematocrit. We know from animal studies that storage of RBCs leads to biochemical and physical changes that hinder their function during transfusion (22-24) and decrease functional capillary density (25). For the depleted RBC 2,3-diphosphateglycerate acid (2, 3 DPG) and ATP concentrations at least one day is required until 50 to
$70 \%$ of the normal level of 2,3 DPG is recovered and $1 \mathrm{wk}$ for full recovery. In the previously mentioned study Nadeau did not observe a decline in vessel density in all of the anemic subjects and speculates that decreased vessel density might offer information about adaptive processes and the pathophysiological changes caused by anemia. In our transfusion study, $\mathrm{Hb}$ levels were very low at the start and obviously increased significantly after the 4-h transfusion. In the current study, $\mathrm{Hb}$ levels decreased slowly over the $4 \mathrm{wk}$ mostly due to anemia of prematurity and iatrogenic blood loss. Adaptive processes are very different in these situations, in particular concerning transfusion where adult RBC of various age are infused. Using a different technology near-infrared photoplethysmography (NIRP) we have previously shown, that elective transfusions do not change the blood flow in arteriovenous anastomoses as the nonnutrient, high volume, low resistance pathway (26). These vessels are the main source for the NIRP signal. Capillary hematocrit is regulated and maintained at comparatively low values which are about $50 \%$ of normal systemic value $(27,28)$. Neonatal RBCs and microcirculatory blood flow differ significantly from the adult. Neonatal RBCs are larger with higher relative viscosity than adult RBCs, whereas blood viscosity is $12 \%$ less in neonates than in adults (29). Neonatal blood also has a larger decrease in blood viscosity in artificial tubes with diameters $<500 \mu \mathrm{m}$ than adult blood. Zilow and Linderkamp (30) showed that both the large cell volume and the increased membrane elasticity of neonatal RBCs contribute to the enhanced reduction of viscosity in narrow tubes. The greater viscosity reduction of neonatal RBCs in narrow vessels may be an important prerequisite for the lower vascular resistance and high flow conditions in the neonate. This might also explain why we found a significant correlation between $\mathrm{Hb}$ levels and FSVD in our patients with a decrease in FSVD in neonates with severe anemia. The importance of blood viscosity to maintain tissue perfusion at the capillary level has been shown in extreme hemodilution with low- and highviscosity solution in the animal model (31).

Both FSVD and incubator temperature decreased over time (Fig. 1). The close correlation of incubator temperature and FSVD (Fig. 2) could be due to maturation of the skin with fewer visible vessels and therefore lower heat loss. It is less likely that the lower incubator temperatures lead to constriction of vessels to reduce heat loss, since incubator temperatures were closely regulated to keep body temperature constant at $37^{\circ} \mathrm{C}$. With NIRP we have found previously, that raising incubator temperature to "comfort temperature" with a temperature difference of less than $2^{\circ} \mathrm{C}$ between abdominal wall and extremities significantly changes skin blood flow in the extremities of premature infants with clinical signs of compromised microcirculation, but not in infants without such signs (32). NIRP primarily assesses the nutritive flow in the deeper layers of the skin. Incubator temperatures in that study were much higher at $38^{\circ} \mathrm{C}$ and we did not find any changes in infants without compromised microcirculation.

With the Cytoscan ${ }^{\mathrm{TM}} \mathrm{A} / \mathrm{R}$, the diameter is measured as the width of the RBC column present in the vessel. The true anatomical vessel diameter therefore could be underestimated. Harris et al.(17) have shown, that identical vessels measured 
by OPS imaging are 4 to $5 \mu \mathrm{m}$ smaller in comparison to intravital fluorescence microscopy. Diameters of the vessels measured in our study ranged from 7 to $24 \mu \mathrm{m}$. In neonates, vessels larger than 25 to $30 \mu \mathrm{m}$ are deeper in the dermis and only small loops can be observed before the vessel disappears into the deeper layer $(33,34)$. In neonates due to the small size of the vessel, arterioles, capillaries, and post capillary venules cannot be clearly distinguished $(8,9)$.

The marginal decrease of RBC vel during the first month of life did not reach statistical significance but RBC vel correlated directly with the $\mathrm{Hb}$ levels. In addition, RBC vel inversely correlated with mean systolic blood pressure. This seems surprising since past studies have shown that blood pressure in premature infants correlates with blood flow (35). In those studies, blood flow was measured with venous occlusion plethysmography, which will determine arterial blood flow in vessels of all sizes. Our RBC vel was obtained in the microvasculature where in neonates arterioles and venules cannot be distinguished with this method. Therefore, our values probably are a mixture of venous and arterial flow velocities. Previous studies have shown that resistance and viscosity play a crucial role in neonatal peripheral blood flow $(35,36)$. Both undergo marked changes in the first days of life. Our values are similar to the results of Norman, who determined $\mathrm{RBC}$ vels in the nail fold capillaries of term infants in the first week of life.

FSVD is used as a measure of tissue perfusion. Because only RBC-perfused vessels are counted, it is also an indirect measure of the oxygen delivery. FSVD significantly decreased possible due to the drop of $\mathrm{Hb}$ levels and or due to maturation effects of skin, which lead to decreased incubator temperatures. From the time of birth, the $\mathrm{Hb}$ concentration is reduced by physiologic reduction of the red blood cells due to anemia of prematurity and iatrogenic blood loss (37). FSVD inversely correlated with heart rate and systolic blood pressure.

In our study, we compared very preterm infants (gestational age $<26 \mathrm{wk}$ ) with preterm neonates (gestational age $>28 \mathrm{wk}$ ). We did not find any differences between the two groups in the first month of life with regard to diameter, red blood cell velocity and vessel density.

OPS imaging allows direct observation of RBC rheology in vivo and enables monitoring of the microcirculatory response to therapy and changes in the microcirculation for example in pathologic conditions such as anemia or infection, thus becoming a valuable clinical tool. This, however, will require a standardized and automated analysis routine that has been validated as a true measure of tissue perfusion. At present, the calculations have to be done off-line and are rather timeconsuming, which limits the current clinical use of OPS imaging. Another problem of OPS imaging for microvascular monitoring in humans is the variability of the vessels measured. Identical vessels cannot be examined over time because it is not possible to identify the same vessel yet. $\mathrm{RBC}$ velocity in particular is difficult to measure since it depends on at least $10 \mathrm{~s}$ video sequences with no movement and vessels of enough length.

In conclusion, images of the microvasulature of the skin can be obtained in premature infants for at least the first month of life. We suggest that OPS imaging can be used to monitor microcirculatory parameters in preterm infants regardless of gestational age or postnatal age. This method allows the assessment of the quality of tissue perfusion for the skin. We know blood pressure homeostasis is superior to maintenance of peripheral perfusion in the newborn period, since this patient population respond to acute hypovolemia with marked decrease in peripheral flow and an increase in peripheral resistance and only small changes in blood pressure (35). Thus, the measurements of the microcirculation of the skin might be an easily accessible window to obtain better understanding of circulatory changes in infants in the postnatal period. Our data are essential as basis for further studies in this field. $\mathrm{Hb}$ levels have a substantial influence on FSVD and therefore need to be taken in account. The surrounding environment, such as incubator temperatures, may also affect the microcirculation and possibly maturation effects of the skin.

\section{REFERENCES}

1. Ellis CG, Jagger J, Sharpe M 2005 The microcirculation as a functional system. Crit Care 9:S3-S8

2. Ince C 2002 The microcirculation unveiled. Am J Respir Crit Care Med 166:1-2

3. Ince C 2004 Microcirculation in distress: a new resuscitation end point? Crit Care Med 32:1963-1964

4. Ince C 2005 The microcirculation is the motor of sepsis. Crit Care 9:S13-S19

5. Boerma EC, Mathura KR, van der Voort PH, Spronk PE, Ince C 2005 Quantifying bedside-derived imaging of microcirculatory abnormalities in septic patients: a prospective validation study. Crit Care 9:R601-R606

6. De Backer D, Creteur J, Preiser JC, Dubois MJ, Vincent JL 2002 Microvascular blood flow is altered in patients with sepsis. Am J Respir Crit Care Med 166:98-104

7. Sakr Y, Dubois MJ, De Backer D, Creteur J, Vincent JL 2004 Persistent microcirculatory alterations are associated with organ failure and death in patients with septic shock. Crit Care Med 32:1825-1831

8. Genzel-Boroviczény O, Stroetgen J, Harris AG, Messmer K, Christ F 2002 Orthogonal Polarization Spectral Imaging (OPS): a novel method to measure the microcirculation in term and preterm infants transcutaneously. Pediatr Res 51:386-391

9. Genzel-Boroviczény O, Christ F, Glas V 2004 Blood transfusion increases functional capillary density in the skin of anemic preterm infants. Pediatr Res 56:751755

10. Harpin VA, Rutter N 1982 Development of emotional sweating in the newborn infant. Arch Dis Child 57:691-695

11. Rutter N 2000 The dermis. Semin Neonatol 5:297-302

12. Lindert J, Werner J, Redlin M, Kuppe H, Habazettl H, Pries AR 2002 OPS imaging of human microcirculation: a short technical report. J Vasc Res 39:368-372

13. Chu DH, Loomis CA 2004 Structure and development of the skin and cutaneous appendages. In: Polin RA, Fox WW, Abman SH (eds) Fetal and Neonatal Physiology. Saunders, Philadelphia, pp 589-596

14. Ryan TJ 1998 Development of the cutaneous circulation. In: Polin RA, Fox WW (eds) Pathophysiology of the Fetus and Neonate. W.B. Saunders Company, Philadelphia, pp 752-761

15. Nolte D, Zeintl H, Steinbauer M, Pickelmann S, Messmer K 1995 Functional capillary density: an indicator of tissue perfusion? Int J Microcirc Clin Exp 15:244249

16. Groner W, Winkelman JW, Harris AG, Ince C, Bouma GJ, Messmer K, Nadeau RG 1999 Orthogonal polarization spectral imaging: a new method for study of the microcirculation. Nat Med 5:1209-1212

17. Harris AG, Sinitsina I, Messmer K 2000 The Cytoscan Model E-II, a new reflectance microscope for intravital microscopy: comparison with the standard fluorescence method. J Vasc Res 37:469-476

18. Harris AG, Sinitsina I, Messmer K 2000 Quantitative analysis of Orthogonal Polarization Spectral images: validation in the hamster dorsal skinfold chamber. In: Messmer, K. (eds) Progress in Applied Microcirculation. Basel: Karger, pp 21-31

19. Langer S, Harris AG, Biberthaler P, von Dobschuetz E, Messmer K 2001 Orthogonal Polarization Spectral Imaging as a tool for the assessment of hepatic microcirculation. A validation study. Transplantation 71:1249-1256

20. Dobbe JG, Streekstra GJ, Atasever B, van Zijrderveld R, Ince C 2008 Measurement of functional microcirculatory geometry and velocity distributions using automated image analysis. Med Biol Eng Comput 46:659-670

21. Nadeau RG, Groner W 2001 The role of a new noninvasive imaging technology in the diagnosis of anemia. J Nutr 131:1610S-1614S

22. Solheim BG, Flesland O, Seghatchian J, Brosstad F 2004 Clinical implications of red blood cell and platelet storage lesions: an overview. Transfus Apher Sci 31:185-189

23. Koch CG, Li L, Sessler DI, Figueroa P, Hoeltge GA, Mihaljevic T, Blackstone EH 2008 Duration of Red-Cell Storage and Complications after Cardiac Surgery. N Engl J Med 358:1229-1239 
24. Adamson JW 2008 New blood, old blood, or no blood? N Engl J Med 358:12951296

25. Tsai AG, Cabrales P, Intaglietta M 2004 Microvascular perfusion upon exchange transfusion with stored red blood cells in normovolemic anemic conditions. Transfusion 44:1626-1634

26. Seidl T, Genzel B, Abicht JM, Christ F 2004 Does red blood cell transfusion change the near infra red photoplethysmography signal in infants? Intensive Care Med 30:1602-1606

27. Mirhashemi S, Messmer K, Afors KE, Intaglietta M 1987 Microcirculatory effects of normovolemic hemodilution in skeletal muscle. Int J Microcirc Clin Exp 6:359-369

28. Tsai AG, Arfors KE, Intaglietta M 1991 Spatial distribution of red blood cells in individual skeletal muscle capillaries during extreme hemodilution. Int J Microcirc Clin Exp 10:317-334

29. Stadler A, Linderkamp O 1989 Flow behavior of neonatal and adult erythrocytes in narrow capillaries. Microvasc Res 37:267-279

30. Zilow EP, Linderkamp O 1989 Viscosity reduction of red blood cells from preterm and full-term neonates and adults in narrow tubes (Fahraeus-Lindqvist effect). Pediatr Res 25:595-599
31. Cabrales P, Tsai AG, Intaglietta M 2004 Microvascular pressure and functional capillary density in extreme hemodilution with low- and high-viscosity dextran and a low-viscosity Hb-based O2 carrier. Am J Physiol Heart Circ Physiol 287:H363$\mathrm{H} 373$

32. Genzel-Boroviczeny O, Seidl T, Rieger-Fackeldey E, Abicht J, Christ F 2007 Impaired microvascular perfusion improves with increased incubator temperature in preterm infants. Pediatr Res 61:239-242

33. Vincent JL, De Backer D 2005 Microvascular dysfunction as a cause of organ dysfunction in severe sepsis. Crit Care 9:S9-S12

34. Tsai AG, Johnson PC, Intaglietta M 2003 Oxygen gradients in the microcirculation Physiol Rev 83:933-963

35. Linderkamp O, Strohhacker I, Versmold HT, Klose H, Riegel KP, Betke K 1978 Peripheral circulation in the newborn: interaction of peripheral blood flow, blood pressure, blood volume, and blood viscosity. Eur J Pediatr 129:73-81

36. Wu PY, Wong WH, Guerra G, Miranda R, Godoy RR, Preston B, Schoentgen S, Levan NE 1980 Peripheral blood flow in the neonate; 1. Changes in total, skin, and muscle blood flow with gestational and postnatal age. Pediatr Res 14:1374-1378

37. Westkamp E, Soditt V, Adrian S, Bohnhorst B, Groneck P, Poets CF 2002 Blood transfusion in anemic infants with apnea of prematurity. Biol Neonate 82:228-232 